\title{
A young non-smoker with high ADA pleural effusion. It's not always tuberculosis
}

\author{
Gopal Chawla', Amrit Pal Kansal'2, Kunal Deokar' \\ ${ }^{1}$ All India Institute of Medical Sciences, Jodhpur, Rajasthan, India \\ ${ }^{2}$ Government Medical College, Patiala, India
}

Lung cancer is posing an ever-increasing medical and social problem due to its increasing morbidity and mortality. Here, we report a case of a young male who was originally being treated for a tubercular pleural effusion but was ultimately diagnosed with non-small cell carcinoma. While considering the diagnosis of pleural effusion in a country like India, where tuberculosis (TB) is endemic, it is not surprising that many clinicians prefer to consider pulmonary tuberculosis ahead of malignancy in the differential diagnosis, especially in a young patient.

A 26-year-old non-smoking and immunocompetent male presented with complaints of an intermittent fever, cough with mild expectoration, right-sided chest pain which increased on deep inspiration, and dyspnea for two months. His routine blood investigations were within normal limits and a general physical examination was also normal. His chest X-ray revealed a pleural effusion with left lower zone heterogeneous infiltration. He was started on anti-tubercular therapy on the basis of a lymphocytic and high ADA (67.7 IU) pleural effusion. However, even after one month, he did not respond to the treatment. Repeated diagnostic thoracentesis showed small and large clusters of malignant squamous epithelial cells exhibiting an increased nuclear-cytoplasmic ratio and nuclear hyperchromatism on MGG and PAP stained cytological smears. IHC was positive for p63 suggesting squamous cell carcinoma with the primary location possibly being in the lung. CECT showed a mass measuring $4 \times 6 \mathrm{~cm}$ in the right lower lobe with a bronchus cut-off sign along with a mild pleural effusion and mediastinal lymphadenopathy (Figure 1). On bronchoscopy, multiple nodules were seen in the right lower lobe bronchus. Endobronchial lung biopsy confirmed the diagnosis of squamous cell carcinoma. The patient was diagnosed with a pT2bN1M1a stage IV squamous cell carcinoma according to the International Union Against Cancer staging system.

Lung cancer in young people is uncommon. It has been reported that only $1.2 \%$ to $2.7 \%$ of patients diagnosed with lung cancer are less than 40 years of age. In the younger group, women are more highly represented than in the older group. Adenocarcinomas are more frequent than squamous cell carcinomas among young and newer smokers [1].

Cigarette smoking is a major risk factor for lung cancer in young people. Factors other than smoking include environmental(second-hand) tobacco smoke, indoor air pollution (e.g. coal and cooking fumes), a family history of lung cancer, occupational and environmental agents (e.g. radon, asbestos, and heavy metals), preexisting lung diseases, and/or human papillomavirus infection [2]. The young man here, however, had not been significantly exposed to any of these factors. Therefore, other factors such as environmental carcinogens or genetic susceptibility might have contributed to the development of lung cancer.

Young patients with lung cancer often have advanced stages at presentation, and this was similar to what we witnessed in our present case. It has been suggested that this is due to the high malignant nature and/or the delayed diagnosis resulting from a low degree of suspicion of cancer, but this relationship is not significant [1]. It is speculated that younger patients can be managed in a more aggressive fashion than older patients because of their better overall medical conditions [2].

It is unknown why younger patients with carcinoma more commonly present with advanced disease. Because bronchogenic carcinoma in this age group is rare, both public and professional awareness is limited. Young patients may not suspect serious illness and often ignore any type of symptoms. Moreover, physicians often may not suspect an underlying carcinoma despite persistent pulmonary symptoms or abnormal findings on chest roentgenograms. The mean duration of symptoms before diagnosis is usually 4-5 months [3].

Therefore, repeated cytology should be performed in cases of pleural effusion that are not responsive to treatment even if the first cytology sample is negative and excludes the possibility of lung cancer. If non-small cell bronchogenic carcinoma is diagnosed and signs of distant metastasis are lacking, further exploration is

Address for correspondence: Gopal Chawla, All India Institute of Medical Sciences, Jodhpur, Rajasthan, India; e-mail: dr.gopalchawla@gmail.com DOI: 10.5603/ARM.a2020.0144

Received: 29.03.2020

Copyright (C) 2020 PTChP

ISSN 2451-4934

Conflict of interest: None declared 

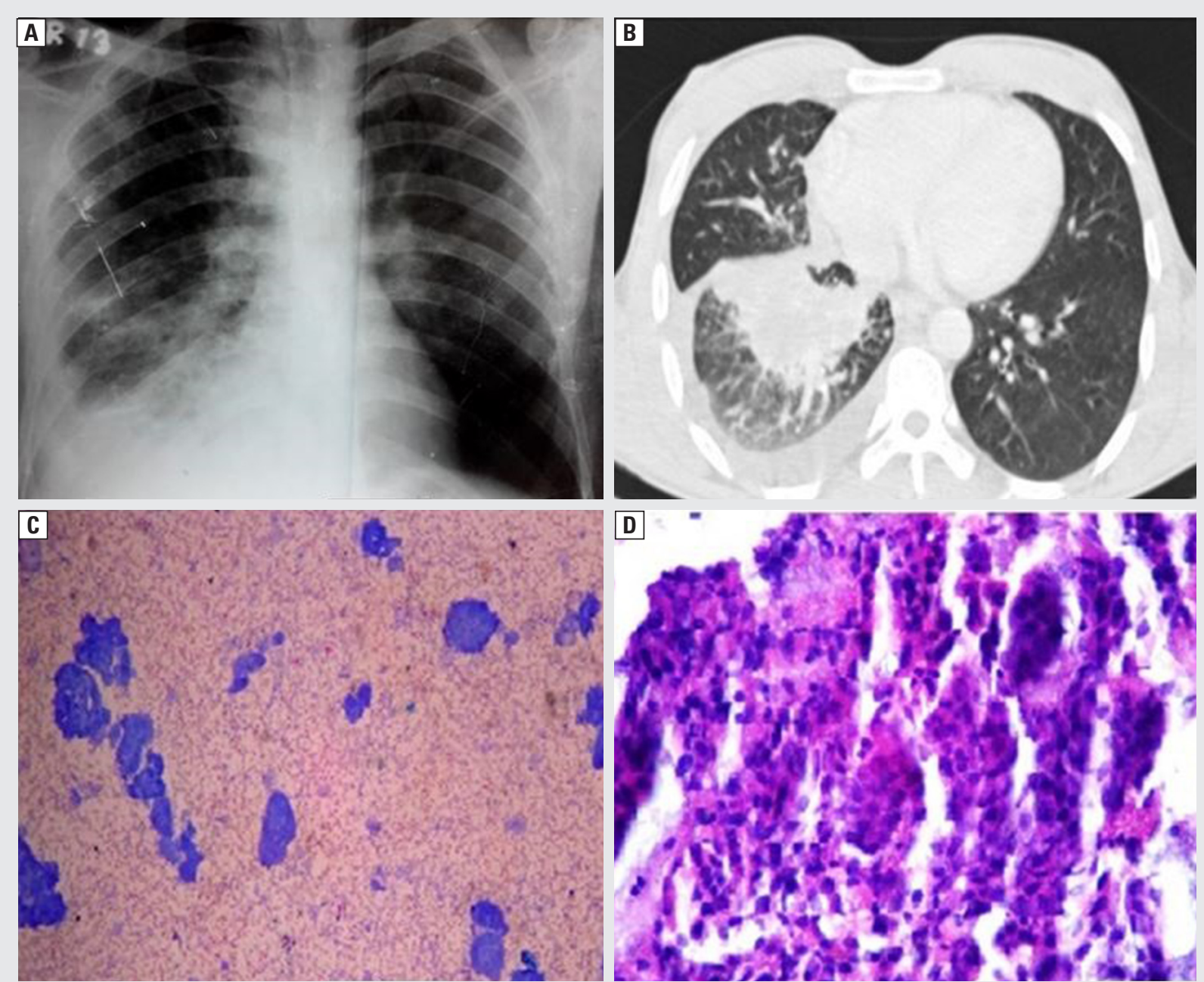

Figure 1. A. Chest X-ray showing a right pleural effusion with atelectasis; B. CT scan showing a mass lesion with pleural effusion; C. Microscopic $10 \times$ picture of the pleural effusion with small and large clusters of atypical cells; $\mathbf{D}$. Endobronchial biopsy $10 \times$ showing atypical cells

necessary because improved survival depends on surgical resection. Because the rarity of the disease in this particular age group often delays the diagnosis, it is very important for all clinicians to consider the possibility of lung cancer in young patients where presentation or follow-up is atypical. Suspicion along with aggressive treatment may lead to an earlier diagnosis and a better prognosis.

\section{References:}

1. Ramalingam S, Pawlish K, Gadgeel S, et al. Lung cancer in young patients: analysis of a surveillance, epidemiology, and end results database. J Clin Oncol. 1998; 16(2): 651-657, doi: 10.1200/JCO.1998.16.2.651, indexed in Pubmed: 9469354.

2. Sekine I, Nishiwaki Y, Yokose T, et al. Young lung cancer patients in Japan: different characteristics between the sexes. Ann Thorac Surg. 1999; 67(5): 1451-1455, doi: 10.1016/s0003-4975(99)00171-x, indexed in Pubmed: 10355430.

3. Subramanian J, Govindan R. Lung cancer in never smokers: a review. J Clin Oncol. 2007; 25(5): 561-570, doi: 10.1200/ ICO.2006.06.8015, indexed in Pubmed: 17290066. 